\title{
Effects of Sustained Inflation or Positive Pressure Ventilation on the Release of Adrenomedullin in Preterm Infants with Respiratory Failure at Birth
}

\author{
Azzurra La Verde, MD ${ }^{1}$ Simone Franchini, MD $\quad$ Giuseppe Lapergola, MD ${ }^{2}$ Gianluca Lista, MD, PhD ${ }^{1}$ \\ Ignazio Barbagallo, MSc, $\mathrm{PhD}^{3}$ Giovanni Livolti, MD, $\mathrm{PhD}^{3}$ Diego Gazzolo, MD, $\mathrm{PhD}^{2,4}$ \\ ${ }^{1}$ Neonatal Intensive Care Unit, V. Buzzi Children's Hospital, ASST-FBF- \\ Sacco, Milan, Italy \\ 2 Neonatal Intensive Care Unit, G. D’Annunzio University of Chieti, \\ Chieti, Italy \\ ${ }^{3}$ Department of Biomedical and Biotechnological Sciences Section of \\ Address for correspondence Diego Gazzolo, MD, PhD, Department of \\ Maternal Fetal and Neonatal Medicine, C. Arrigo Children's Hospital, \\ Spalto Marengo 46, I-15100 Alessandria, Italy \\ (e-mail: dgazzolo@hotmail.com). \\ Biochemistry, University of Catania, Catania, Italy \\ ${ }^{4}$ Department of Maternal Fetal and Neonatal Medicine, C. Arrigo \\ Children's Hospital Alessandria, Alessandria, Italy
}

Am J Perinatol 2019;36(suppl S2):S110-S114.
Abstract
Objective Delivery room (DR) management may play an important role in the development and prevention of lung injury. Therefore, in a cohort of low birth weight infants (LBW), we investigated the effects of two different lung recruitment maneu- vers, such as positive pressure ventilation (PPV) and sustained inflation (SI) on adrenomedullin (AM), a well-established lung-specific vasoactive agent.
Study Design This is a prospective case-control randomized study in 44 LBW infants spontaneously breathing with respiratory failure at birth requiring respiratory support. LBW were randomized to receive PPV $(n=22)$ or SI $(n=22)$ support. AM was
Keywords
- positive pressure ventilation
- sustained lung inflation
- adrenomedullin
- respiratory failure
- resuscitation
- preterm infants measured from blood in samples collected at birth from arterial artery (BLT0) and at 1-hour (BLT1) and at 24-hour (BLT2) from peripheral venous site. AM assessment in urine samples was performed at 1-hour (URT1) and at 24-hour (URT2).
Results No significant differences in AM $(p>0.05)$ blood $(\mathrm{T} 0-\mathrm{T} 2)$ and urine $(\mathrm{T} 1, \mathrm{~T} 2)$ levels were observed between groups.
Conclusion The present data, showing the absence of any differences in AM blood and urine levels, suggest that PPV and SI are both feasible and equally effective DR maneuvers. The findings open the way to further studies evaluating the effects of PPV and SI on short-/long-term respiratory outcome through biomarkers assessment.

There is growing evidence that the delivery room (DR) management may play an important role in the development and prevention of lung injury, as well as an improper support can cause lung damage. ${ }^{1}$ In humans, it has been demonstrated that the lung recruitment maneuver, namely, sustained inflation (SI) can facilitate cardiorespiratory transition. ${ }^{2}$ In fact, SI when compared with positive pressure ventilation (PPV) reduced the need of intubation while no benefits have been shown on the length of respiratory support and the incidences of bronchopulmonary dysplasia
(BPD) and death. ${ }^{2}$ In this regard, the measurement of quantitative parameters, such as lung-specific vasoactive agents, could be particularly useful to monitor the effectiveness of DR maneuvers.

Adrenomedullin (AM) is a, 52-amino acid, vasoactive peptide first isolated from human pheochromocytoma which belongs to the calcitonin family of peptides comprising calcitonin, calcitonine gene related peptide (CGRP), and amylin and intermedin. ${ }^{3}$ Cultured vascular endothelial cells and smooth muscle cells produce AM, which elicits its
Copyright $\odot 2019$ by Thieme Medical Publishers, Inc., 333 Seventh Avenue, New York, NY 10001, USA. Tel: +1(212) 584-4662. ISSN 0735-1631. 
vasodilator activity with a reduction of lung endothelial hyperpermeability, through an increase of intracellular c-adenosin monophophate. ${ }^{3} \mathrm{AM}$ has been also shown to be involved in the regulation of systemic and pulmonary blood flow during neonatal cardiovascular adaptation and during birth stress. ${ }^{4}$

Therefore, the aim of the present study was to investigate, in a cohort of preterm infants complicated by respiratory failure at birth, the effects of two resuscitation maneuvers, such as SI or PPV on AM release in biological fluids.

\section{Materials and Methods}

We conducted a prospective case-control randomized study in a cohort of 70 low-birth-weight (LBW) infants of low gestational age (GA; $28^{0 / 7}-30^{6 / 7}$ weeks), at risk for respiratory distress syndrome (RDS) recruited at our third level referral center for neonatal intensive care (NICU), from March 2013 to October 2014. Infants were assigned to receive either SI $(n=22)$ or PPV $(n=22)$ maneuvers (1SI:1PPV) (-Fig. 1).

Approval was obtained from the local ethic committees and parents gave informed and signed consent before the inclusion in the study.

Exclusion criteria were (1) infants with adequate respiratory effort and heart rate $(\mathrm{HR})>100$ beats per minute (bpm) or requiring only initial nasal continuous positive airway pressure (NCPAP), (2) infants requiring resuscitation man- euvers and intubation, (3) presence of congenital heart, cerebral, lung and abdominal malformations, and (4) need of noninvasive ventilation or intubation in DR and fetal hydrops.

\section{Delivery Room Management}

After birth and cord clamping, infants were placed under a radiant heat source, in a plastic wrap, positioning the head in a "sniffing" position to open the airway, clearing the airway if necessary with suction catheter, drying the baby, and stimulating breathing if necessary.

In spontaneously breathing infants with apnea or gasping or $\mathrm{HR}<100 \mathrm{bpm}$, an initial NCPAP of $5 \mathrm{~cm} \mathrm{H}_{2} \mathrm{O}$ facial mask was applied for 30 seconds for the initial stabilization. In case of persistent apnea/gasping, or of a $\mathrm{HR}<100 \mathrm{bpm}$ after administering NCPAP, two different resuscitation strategies (SI or PPV) by face mask were performed to manage RDS.

In both groups, neonatal care was started with a fraction of inspired oxygen $\left(\mathrm{FiO}_{2}\right)$ of 0.30 then titrated to maintain arterial oxygen saturation $\left(\mathrm{SaO}_{2}\right)$ level of 85 to $95 \%$ at 10 minutes from birth. All enrolled infants were transferred to the NICU with NCPAP support of $5 \mathrm{~cm} \mathrm{H}_{2} \mathrm{O}$.

\section{Sustained Inflation}

In $\mathrm{SI}$ infants, a pressure-controlled $\left(25 \mathrm{~cm} \mathrm{H}_{2} \mathrm{O}\right)$ inflation was sustained for 15 seconds, followed by the delivery of $5 \mathrm{~cm}$ $\mathrm{H}_{2} \mathrm{O}$ NCPAP, using a neonatal mask and a T-piece ventilator (Neopuff Infant T-Piece Resuscitator, Fisher \& Paykel,

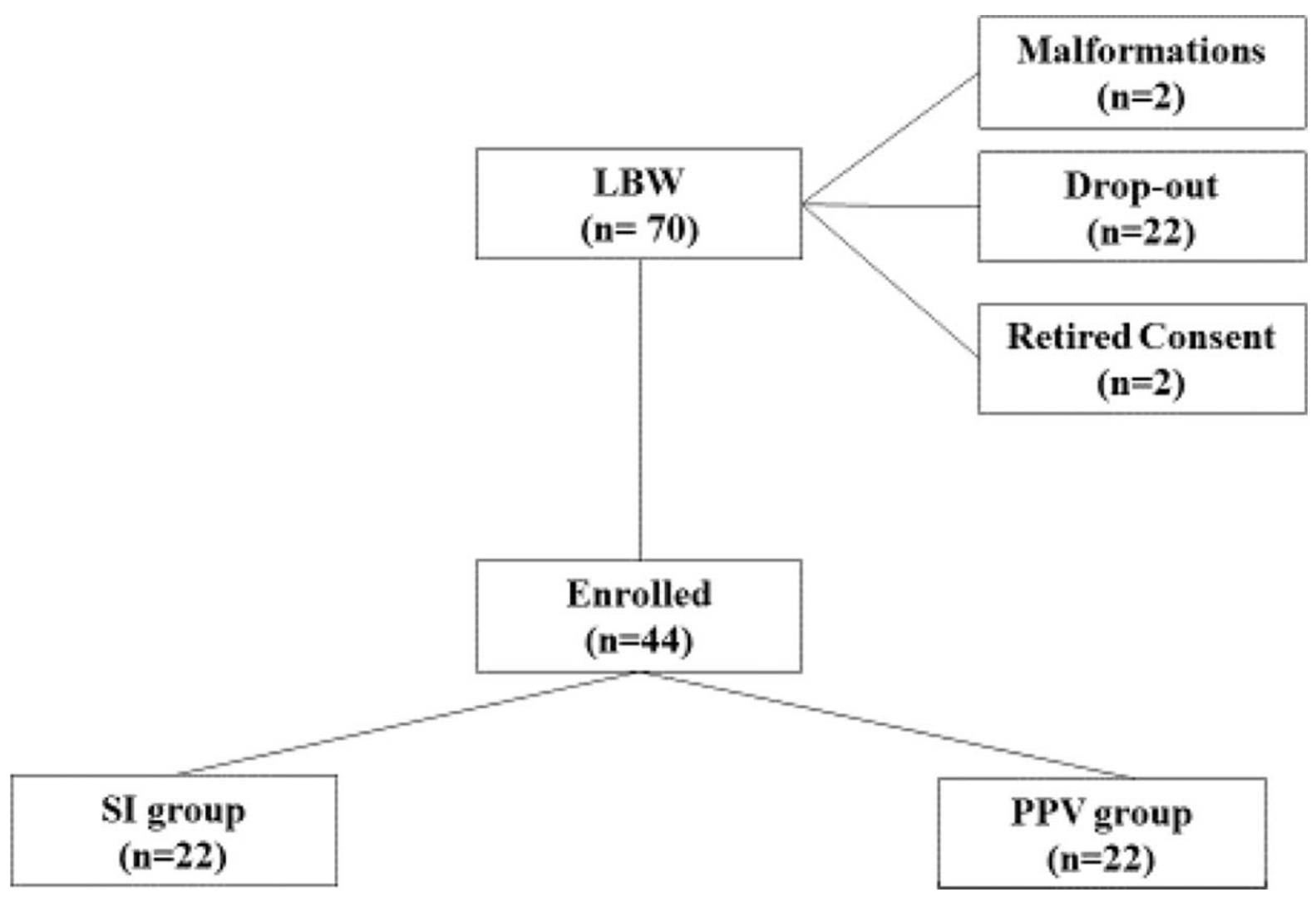

Fig. 1 Flow chart. 
Auckland, New Zealand). ${ }^{2}$ Thereafter, cardiorespiratory activity was observed for 6 to 10 seconds; if respiratory symptoms persisted and/or the HR was $>60$ and $<100$ bpm despite NCPAP, SI maneuver was performed.

\section{Positive Pressure Ventilation}

PPV with a peak pressure of $25 \mathrm{~cm} \mathrm{H}_{2} \mathrm{O}$, a positive endexpiratory pressure of $5 \mathrm{~cm} \mathrm{H}_{2} \mathrm{O}$ and with assisted ventilation rates of 40 to 60 breaths per minute was applied ${ }^{2}$ and maintained until the achievement/stabilization of a HR $>100$ bpm or spontaneous effective breaths.

\section{Monitoring Parameters}

Newborns were monitored using pre-ductal $\mathrm{SaO}_{2}$ (Radical 8 pulse oximeter, Masimo Corporation, Irvine, CA). For each infant, we recorded GA, birth weight, gender, twin occurrences, main maternal pregnancy diseases, prenatal steroid course, delivery mode, Apgar's scores at 1 and 5 minutes, and $\mathrm{FiO}_{2}$ support. We also recorded the incidence of: pneumothorax (PNX), (BPD), ${ }^{5}$ early neonatal death, intraventricular hemorrhage (IVH), ${ }^{6}$ periventricular leukomalacia (PVL); patent ductus arteriosus (PDA) requiring surgical closure, postnatal glucocorticoids administration, retinopathy of prematurity (ROP), ${ }^{7}$ surfactant administration, NCPAP failure $<72$-hour, and the need of mechanical ventilation (MV).

\section{Adrenomedullin Measurement}

Blood and urine samples ( $1 \mathrm{~mL})$, for AM measurement, were collected at scheduled monitoring time-points: before resuscitation maneuvers, at birth, from the arterial umbilical cord (BLT0); at 1-hour (BLT1) and at 24-hour from birth (BLT2) from peripheral blood. Identically, urine samples were collected at 1-hour (URT1) and at 24-hour (URT2) from birth.

AM was measured by a specific radio-immunometric assay kit (Phoenix Pharmaceuticals, Mountain View, CA). The sensitivity was $0.34 \mathrm{fmol} / \mathrm{mL}$; the intra-interassay coefficients of variance were 5.1 and $12.0 \%$, respectively.

\section{Statistical Analysis}

To calculate the sample size, we used AM concentration changes during transition phase as the main parameter. As no basic data are available for such specific population, we assumed a decrease in standard deviation (SD) of 0.5 in AM to be clinically significant. Indeed, considering a $\alpha=0.05$ and using a two-sided test, we estimated a power of 0.70 , recruiting 44 infants in whom SI and PPV procedures were performed. The sample size was calculated by using nQuery Advisor (Statistical Solutions, Saugus, MA), version 5.0.

Results were expressed as mean and SD for continuous variables and absolute and relative frequencies for categorical variables. AM concentrations were expressed as median and interquartile ranges. Parameters of the two groups were compared using Student's t-test or two-sided MannWhitney $U$-test, when the data were not normally distributed for continuous variables, and Chi-square or Fisher's exact test for categorical variables. Normality of distribution was determined using the Kolmogorov-Smirnov test. Com- parisons of $\mathrm{AM}$ at the different monitoring time-points were performed by Wilcoxon's test. The significance threshold was $p<0.05$.

\section{Results}

-Table 1 reports perinatal characteristics in the studied groups. No significant $(p>0.05)$ differences were observed regarding GA, birth weight, the incidence of gender, small gestational age (SGA), twins, chorioamnionitis, prolonged premature rupture of membrane (pPROM), preeclampsia, prenatal steroid course administration, and caesarean section ( $p>0.05)$ between groups. At birth, Apgar scores at 1 and 5 minutes and $\mathrm{FiO}_{2}$ administration were superimposable ( $p>0.05$ ) between groups.

-Table 2 reports the main neonatal outcomes in the studied groups. No significant $(p>0.05)$ differences between groups were observed in the incidence of PNX, BPD, early neonatal death, IVH, PVL, PDA, postnatal glucocorticoids administration, ROP, surfactant administration, NCPAP failure $<72$-hour, the need of MV, and the length of respiratory support.

In the SI group, AM pattern was characterized by a significant $(p<0.05)$ increase in AM blood levels from BLT0 to BLT1 (1 hour) with a significant decrease $(p<0.05)$ at BLT2 (24-hour). No difference $(p>0.05)$ has been found between BLT0 and BLT2. AM urine levels were superimposable ( $p>0.05$ ) at URT1 to URT2 (- Fig. 2).

Table 1 Perinatal characteristics infants supported by sustained inflation (SI) and positive pressure ventilation (PPV)

\begin{tabular}{|l|l|l|}
\hline Parameters & $\begin{array}{l}\text { SI } \\
(\boldsymbol{n}=\mathbf{2 2})\end{array}$ & $\begin{array}{l}\text { PPV } \\
(\boldsymbol{n}=\mathbf{2 2})\end{array}$ \\
\hline GA (wk) & $30 \pm 1$ & $30 \pm 1$ \\
\hline BW (g) & $1,495 \pm 90$ & $1,520 \pm 80$ \\
\hline Gender (male/female; tot; \%) & $8 / 22(36)$ & $7 / 22(32)$ \\
\hline SGA (tot; \%) & $6 / 22(27)$ & $6 / 22(27)$ \\
\hline Twins (tot; \%) & $7 / 22(32)$ & $6 / 22(26)$ \\
\hline Chorioamnionitis (tot; \%) & $4 / 22(18)$ & $3 / 22(14)$ \\
\hline pPROM (tot; \%) & $7 / 22(32)$ & $6 / 22(27)$ \\
\hline $\begin{array}{l}\text { PIH requiring treatment (tot; } \\
\%)\end{array}$ & $7 / 22(32)$ & $8 / 22(36)$ \\
\hline $\begin{array}{l}\text { Prenatal steroid complete } \\
\text { course (tot; \%) }\end{array}$ & $20 / 22(90)$ & $20 / 22(90)$ \\
\hline Caesarean delivery (tot; \%) & $20 / 22(90)$ & $20 / 22(90)$ \\
\hline Apgar's score at 1 minute & $6 \pm 1$ & $6 \pm 1$ \\
\hline Apgar's score at 5 minute & $8 \pm 1$ & $8 \pm 1$ \\
\hline FiO 2 required in DR & $0.33 \pm 0.5$ & $0.32 \pm 0.5$ \\
\hline
\end{tabular}

Abbreviations: BW: birth weight; DR: delivery room; $\mathrm{FiO}_{2}$ : fraction of inspired oxygen; GA: gestational age; PIH: pregnancy induced hypertension; pPROM: prolonged premature rupture of membrane (>18 h); SGA: small gestational age; tot: total.

Note: Data given as mean \pm SD. 
Table 2 Main outcome measures in infants supported by sustained inflation (SI) and positive pressure ventilation (PPV)

\begin{tabular}{|l|l|l|}
\hline Main outcomes & $\begin{array}{l}\text { SI } \\
(\boldsymbol{n}=\mathbf{2 2})\end{array}$ & $\begin{array}{l}\text { PPV } \\
(\boldsymbol{n}=\mathbf{2 2})\end{array}$ \\
\hline PNX (tot; \%) & $1 / 22(4.5)$ & $0 / 22$ \\
\hline BPD (tot; \%) & $0 / 22$ & $0 / 22$ \\
\hline Death (tot; \%) & $0 / 22$ & $0 / 22$ \\
\hline IVH > II (tot; \%) & $1 / 22(4.5)$ & $1 / 22(4.5)$ \\
\hline PVL (tot; \%) & $2 / 22(9)$ & $1 / 22(4.5)$ \\
\hline PDA (tot; \%) & $0 / 22$ & $0 / 22$ \\
\hline $\begin{array}{l}\text { Postnatal glucocorticoids } \\
\text { (tot; \%) }\end{array}$ & $0 / 22$ & $0 / 22$ \\
\hline ROP > II (tot; \%) & $3 / 22(13.6)$ & $1 / 22(4.5)$ \\
\hline NEC (tot; \%) & $0 / 22$ & $0 / 22$ \\
\hline $\begin{array}{l}\text { Surfactant administration } \\
\text { (tot; \%) }\end{array}$ & $7 / 22(31)$ & $4 / 22(18)$ \\
\hline $\begin{array}{l}\text { NCPAP failure }<\text { 72 hour } \\
\text { (tot; \%) }\end{array}$ & $1 / 22(4.5)$ & $0 / 22$ \\
\hline MV (tot; \%) & $1 / 22(4.5)$ & $0 / 22$ \\
\hline Respiratory support (d) & $3 \pm 1$ & $2.5 \pm 1$ \\
\hline
\end{tabular}

Abbreviations: BPD, bronchopulmonary dysplasia; IVH, intraventricular hemorrhage; MV, mechanical ventilation; NCPAP, nasal continuous positive airway pressure; NEC, necrotizing enterocolitis requiring surgical repair; PDA, patent ductus arteriosus requiring surgical closure; PNX, pneumothorax; PVL, periventricular leukomalacia; ROP, retinopathy of prematurity; tot, total.

In the PPV group, AM pattern of concentration showed a flat trend and no significant $(p>0.05)$ differences in AM blood (BLT0-BLT2) and urine (URT1-URT2) levels have been found.

When AM different biological fluids levels were compared, no significant $(p>0.05)$ differences between SI and PPVgroups were detectable at BLT0 to BLT2 and URT1 to BLT2 ( - Fig. 2).

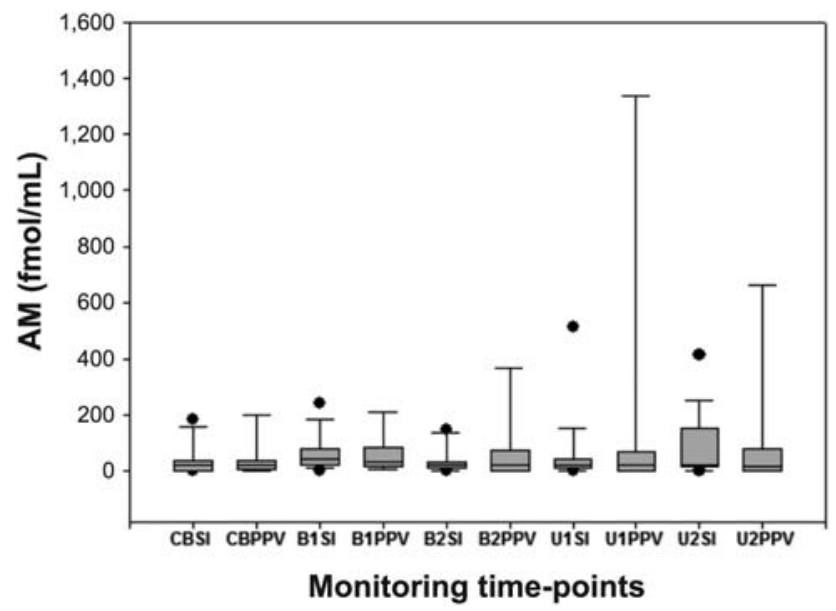

Fig. 2 Adrenomedullin (AM) concentration ( $\mathrm{fmol} / \mathrm{mL}$ ) in blood (B) and urine samples $(\mathrm{U})$ in the sustained lung inflation $(\mathrm{SI})$ and positive pressure ventilation (PPV) groups measured before resuscitation maneuvers at birth from cord blood (CB), at 1 hour from birth (B1-U1) and at 24-hour from birth (B2-U2). Data are given as medians and 5 and 95 centiles.
Since AM might be oxygen-dependent we also calculated blood/urine $\mathrm{FiO}_{2}$ :AM ratio at BLT0 to BLT2 and URT1 to URT2. No significant $\mathrm{FiO}_{2}$ :AM $(p>0.05)$ differences between groups were observed at BLT0-BLT2 and URT1 to URT2.

\section{Discussion}

The DR management is an aspect of respiratory care of preterm infants that has not yet been fully elucidated. ${ }^{1}$ Neonatologists are aware that lung protection should start in the DR to let the high-risk infants to optimize lung draining and recruitment and to avoid damage. ${ }^{1,2}$ To date, the effectiveness of sustained inflation procedure is still under investigation and matter of debate. At this stage, standard clinical, laboratory or monitoring parameters performed in DR did not allow to draw any conclusion. ${ }^{8}$ In this setting, the measurement of biomarkers involved in the so called transitional phase may offer useful information on the effectiveness of DR management. ${ }^{3,4}$

In the present study, we found that blood and urine concentrations of AM, a lung-specific vasoactive agent did not change in the first 24-hour from birth in infants undergone to SI or PPV DR maneuvers. Furthermore, AM blood levels were higher at 1 hour time-point in the SI group when compared with birth and 24-hour time-points.

To the best of our knowledge, this is the first observation comparing a standard DR procedure, such as PPV with SI, by means of AM.

The finding of no differences in AM blood and urine levels between SI and PPV warrants further consideration. In particular, AM is known to: (1) be lung and endothelial cells specific $^{3}$ (2) enhance the anti-inflammatory, antioxidant, and anti-hypoxic defense of many tissues; ( 3 ) be related with acute and chronic lung damage ${ }^{9,10} ;(4)$ increase pulmonary angiogenesis and attenuate alveolar simplification and pulmonary hypertension in animal model ${ }^{9,10}$; and (5) protection against acute lung injury due to different modalities of respiratory support. ${ }^{11}$ Altogether, it is reasonable to infer that AM properties, such as antioxidant, anti-inflammatory, and lung vasoactive agent are not affected independently from PPV or SI DR management. The finding is, of note, bearing in mind that all recruited infants followed the same DR initial management, such as NCPAP. Further investigations aimed at evaluating AM long-term pattern of concentration in biological fluids in wider population are needed. Short-/long-term respiratory follow-up will elucidate whether the early AM blood levels increase in SI group at 1 hour from birth could be expression of a better hemodynamic adaptation to DR procedure. In this respect, the AM role in perinatal adaptation has been reported. Boldt et al showed increased AM levels in birth stress infants due to prolonged vaginal delivery and/or low arterial $\mathrm{pH}^{4}{ }^{4}$ Results were consistent with human and animal model data supporting the physiological role for this peptide in the neonatal vascular adaptation. ${ }^{12,13}$ The mechanisms through which AM acts as vasodilator in the transitional phase are not fully clarified. On one hand, it has been reported that it does not act either by release of a dilator prostaglandin, or 
by stimulating CGRP. ${ }^{3}$ On the other hand, it behaves as an endothelial-dependent vasodilator through the release of nitric oxide; in a sheep-based model AM induced an increase in pulmonary blood flow via nitric oxide release and partly on adenosin tri phosphate channel activation in absence of any involvement of CGRP receptor or a cyclooxygenase-mediated mechanism. ${ }^{14,15}$

In the present study, bearing in mind the powerful AM antihypoxia action we also recorded the $\mathrm{FiO}_{2}$ : $\mathrm{AM}$ in the first 24-hour from birth. The absence of any differences in AM levels in blood and urine fluids in inter-/intragroups deserves further consideration. The pros reside in the possibility of an AM employment as a marker of longitudinal DR management effectiveness. In this regard, taking into account AM short half-life (approximately 1 hour) it is reasonable to gather the potential usefulness of its measurement, especially in the transitional phase. Additional advantages reside in its measurement in less-stressing urine fluid. The cons lie in the inter/intraobserver variability due to different experienced teams in the DR.

\section{Limitations}

Finally, the present study shows some limitations. The main are (1) the small sample size, (2) the study design itself, and (3) the different attitude by the treating physician to perform one or another DR management. We conducted a casecontrol study involving different DR teams, although the protocols were very similar and standardized in our NICUs. Altogether, further multicenter randomized controlled trials (RCTs) will be justified.

\section{Conclusion}

In conclusion, the present data on the absence of any differences in AM between SI and PPV DR management suggest that both are feasible and equally effective. Results open the way to further investigations evaluating the effectiveness of different DR management at short-/long-term respiratory outcome through biomarkers assessment.

\section{Funding}

This study is part of the I.O. PhD International Program, under the auspices of the Italian Society of Neonatology and of the Neonatal Clinical Biochemistry Research Group, and was partially supported by grants to Diego Gazzolo from "I Colori della Vita Foundation," Italy.
Conflict of Interest

None declared.

\section{References}

1 Chawla S, Foglia EE, Kapadia V, Wyckoff MH. Perinatal management: what has been learned through the network? Semin Perinatol 2016;40(06):391-397

2 Lista G, Boni L, Scopesi F, et al; SLI Trial Investigators. Sustained lung inflation at birth for preterm infants: a randomized clinical trial. Pediatrics 2015;135(02):e457-e464

3 Gazzolo D, Abella R, Marinoni E, et al. New markers of neonatal neurology. J Matern Fetal Neonatal Med 2009;22(Suppl 3):57-61

4 Boldt T, Luukkainen P, Fyhrquist F, Pohjavuori M, Andersson S. Birth stress increases adrenomedullin in the newborn. Acta Paediatr 1998;87(01):93-94

5 Jobe AH, Bancalari EH. Controversies about the definition of bronchopulmonary dysplasia at 50 years. Acta Paediatr 2017; 106(05):692-693

6 Papile LA, Burstein J, Burstein R, Koffler H. Incidence and evolution of subependymal and intraventricular hemorrhage: a study of infants with birth weights less than 1,500 gm. J Pediatr 1978;92 (04):529-534

7 International Committee for the Classification of Retinopathy of Prematurity. The international classification of retinopathy of prematurity revisited. Arch Ophthalmol 2005;123(07): 991-999

8 Bruschettini M, O'Donnell CP, Davis PG, et al. Sustained versus standard inflations during neonatal resuscitation to prevent mortality and improve respiratory outcomes. Cochrane Database Syst Rev 2017;7:CD004953

9 Talero E, Di Paola R, Mazzon E, Esposito E, Motilva V, Cuzzocrea S. Anti-inflammatory effects of adrenomedullin on acute lung injury induced by Carrageenan in mice. Mediators Inflamm 2012; 2012:717851

10 Vadivel A, Abozaid S, van Haaften T, et al. Adrenomedullin promotes lung angiogenesis, alveolar development, and repair. Am J Respir Cell Mol Biol 2010;43(02):152-160

11 Müller HC, Witzenrath M, Tschernig T, et al. Adrenomedullin attenuates ventilator-induced lung injury in mice. Thorax 2010; 65(12):1077-1084

12 de Vroomen M, Takahashi Y, Gournay V, Roman C, Rudolph AM, Heymann MA. Adrenomedullin increases pulmonary blood flow in fetal sheep. Pediatr Res 1997;41(4, Pt. 1):493-497

13 Rudolph AM. Adrenomedullin: its role in perinatal adaptation. Acta Paediatr 1998;87(03):235-236

14 Takahashi Y, de Vroomen M, Gournay V, Roman C, Rudolph AM, Heymann MA. Mechanisms of adrenomedullin-induced increase of pulmonary blood flow in fetal sheep. Pediatr Res 1999;45(02): 276-281

15 Nossaman BD, Feng CJ, Kaye AD, et al. Pulmonary vasodilator responses to adrenomedullin are reduced by NOS inhibitors in rats but not in cats. Am J Physiol 1996;270(5 Pt 1):L782-L789

Erratum: Fig. 1 has been updated as per the Erratum published online on July 15, 2020. DOI of the Erratum is $10.1055 / \mathrm{s}-0040-1714104$. 